Differentiation. 2013 October ; 86(3): 121-125. doi:10.1016/j.diff.2013.02.005.

\title{
Collective migration and cell jamming
}

\author{
Sanaz Monirosadat Sadati ${ }^{3}$, Nader Taheri Qazvini ${ }^{1,3}$, Ramaswamy Krishnan ${ }^{2}$, ChanYoung \\ Park $^{3}$, and Jeffrey J. Fredberg ${ }^{3}$ \\ ${ }^{1}$ School of Chemistry, College of Science, University of Tehran, Tehran, Iran \\ ${ }^{2}$ Beth Israel Deaconess Medical Center, Harvard Medical School, Boston, MA 02115 \\ ${ }^{3}$ Harvard School of Public Health, Boston, MA 02115
}

\begin{abstract}
Our traditional physical picture holds with the intuitive notion that each individual cell comprising the cellular collective senses signals or gradients and then mobilizes physical forces in response. Those forces, in turn, drive local cellular motions from which collective cellular migrations emerge. Although it does not account for spontaneous noisy fluctuations that can be quite large, the tacit assumption has been one of linear causality in which systematic local motions, on average, are the shadow of local forces, and these local forces are the shadow of the local signals. New lines of evidence now suggest a rather different physical picture in which dominant mechanical events may not be local, the cascade of mechanical causality may be not so linear, and, surprisingly, the fluctuations may not be noise as much as they are an essential feature of mechanism. Here we argue for a novel synthesis in which fluctuations and non-local cooperative events that typify the cellular collective might be illuminated by the unifying concept of cell jamming. Jamming has the potential to pull together diverse factors that are already known to contribute but previously had been considered for the most part as acting separately and independently. These include cellular crowding, intercellular force transmission, cadherindependent cell-cell adhesion, integrin-dependent cell-substrate adhesion, myosin-dependent motile force and contractility, actin-dependent deformability, proliferation, compression and stretch.
\end{abstract}

\section{Keywords}

Heterogeneity; cooperativity; kinetic arrest; glass transition

\section{Introduction}

Metastasis and invasion, as well as development, remodeling and wound repair, all depend upon collective cellular migration. Rather than moving individually, cells tend to migrate collectively in sheets, ducts, strands and clusters. ${ }^{1-3}$ Collective cellular migration is poorly understood, however, and has been highlighted as being among the 10 greatest unsolved mysteries in all of biology. ${ }^{4}$ Here we begin with consideration of intercellular physical forces and their role in cell biology, which in recent years has come to be called the field of mechanobiology 5 , and then go on to speculate about collective phenomena viewed through a prism borrowed from recent advances in understanding dynamics of inert soft condensed

(C) 2013 Interational Society Of Differentition. Published by Elsevier B.V. All rights reserved.

Publisher's Disclaimer: This is a PDF file of an unedited manuscript that has been accepted for publication. As a service to our customers we are providing this early version of the manuscript. The manuscript will undergo copyediting, typesetting, and review of the resulting proof before it is published in its final citable form. Please note that during the production process errors may be discovered which could affect the content, and all legal disclaimers that apply to the journal pertain. 
matter. In particular, we address dynamic heterogeneity, cooperativity, and kinetic arrest, and then argue for a synthesis of these largely unappreciated properties into a new physical picture. Attention is restricted to the cases of the epithelial or endothelial monolayer.

The traditional reductionist view holds that cooperative cellular events are mediated at the level of the local cell-cell interaction through the agency of a spectrum of physical factors that include cell-generated forces, cell recognition, polarization, selective affinity, and differential adhesion together with gradients of morphogens and phase-gradient encoding of gene oscillations. ${ }^{6-11}$ Cell motility then provides the mechanical agitation that is required for the system to overcome cohesive energy barriers and thus explore various configurational possibilities before ultimately stabilizing into a favorable final state. ${ }^{11}$ Physical forces in question (Fig. 1) include those supported by cytoskeleton (not shown), those exerted across adhesions between the cell base and its substrate (red arrows), and those exerted across each junction between a cell and its immediate neighbors ${ }^{3}$ (blue arrows). Since the time of D'Arcy Thompson ${ }^{12}$ if not earlier, physical forces such as these operating at the cellular level have been recognized as being fundamental to biological form and function, but for almost as long the forces themselves have remained virtually hidden from view.

\section{Dynamic heterogeneity}

To fill that gap, experimental methods recently developed make these hidden forces visible and even resolve forces exerted across the cell-cell junction into distinct normal (tensile) versus shear components. ${ }^{13-19}$ Surprisingly, even in a homogeneous monolayer these measurements reveal dynamic heterogeneities that are striking. Within the monolayer, intercellular forces fluctuate rather severely in space and in time, but are tied neither to any particular position within the monolayer nor to any particular cell. ${ }^{15,17,20}$ The heterogeneity is dynamic, therefore, not structural. ${ }^{17,18,20}$ If at any instant the intercellular tension is mapped in relief across a homogeneous monolayer, the topography is reminiscent of neither the planes of Kansas nor the rolling hills of Vermont as much as the rugged landscape of the Himalayas (Fig. 2). The rugged peaks that define that stress landscape arise from cooperativity across clusters of roughly 10 to 50 cells and thereby account for the cooperative motion of cell packs; over that scale, super-cellular force chains, or force clusters, pull cohesively, coherently, and cooperatively. ${ }^{15,17}$

Because the field of intercellular stress need not be isotropic, an ellipse is sometimes used to represent schematically the local state of cellular stress within the monolayer plane (Fig. 2). In that case local stress anisotropy corresponds to the departure of each ellipse from circularity, where the major axis of each ellipse corresponds to the maximal principal stress, and the minor axis corresponds to the minimal principal stress. Local principal stress orientations are defined by the orientation of each ellipse. Local tension is the sum of the two principal radii of each ellipse. Much as in a weather map, clearly evident in Fig. 2 are strong heterogeneity across the monolayer and strong local cooperativity spanning many cells.

\section{Cooperativity}

While the stress landscape is rugged and the associated heterogeneity is dynamic, certain systematic relationships emerge. In particular, there is a strong tendency for local cellular migration velocity (red arrows, Fig. 2) to follow the local orientation of the maximal principal stress, i.e., the orientation of the stress ellipse. This tendency, called plithotaxis, is a potent mechanism of collective cell guidance and is mediated through the agency of local intercellular stresses exerted between neighboring cells across mutual cell-cells junction. ${ }^{14,15}$ For example, consider the monolayer comprising epithelial breast-cancer 
MCF10A cells ${ }^{15}$, and let $\varphi$ be the angle of the local migration velocity relative to the orientation of the local maximal principal stress, where the distribution of $\varphi$ is represented as a rose of directions (Fig. 3). Averaged over the entire monolayer, the angular distribution of $\varphi$ is clustered strongly around zero degrees, indicating that local principal stresses and local migration velocities are strongly aligned. When MCF10A cells overexpress the oncogene ErbB2/HER-2/neu, which promotes proliferation and leads to even more cellular crowding, the distribution of $\varphi$ becomes even narrower, indicating that plithotaxis has become enhanced and cell guidance has become even stronger. By contrast, when MCF10A cells overexpress the oncogene 14-3-3 $\zeta$, which decreases expression of cell-cell junctional markers, the distribution of $\varphi$ broadens, indicating that plithotaxis has become attenuated and cell guidance has been lost, and much the same loss of cell guidance is caused by calcium chelation or by E-cadherin antibodies. ${ }^{15}$ Together, these observations suggest that plithotaxis rests on cooperativity of mechanical stresses across many cell-to-cell junctions.

\section{Kinetic arrest}

Dynamic heterogeneity and associated cooperativity show interesting dependencies on cellular density and other factors. As cellular density in an expanding monolayer sheet increases as a result of proliferation, and cells therefore become increasingly crowded, cooperative packs become progressively bigger and slower ${ }^{17}$ (Fig. 4). And as cellular crowding approaches some critical threshold, relative motion of neighboring cells slows dramatically and spatial cooperativity of these motions expands. These changes in dynamics need not be accompanied by discernible alteration in cellular structure, however. The basic notion is that with more crowding each cell can become increasingly caged by its neighbors. ${ }^{15,17,20,21}$ And as the effects of caging become progressively stronger, it becomes increasingly difficult for rearrangements amongst neighboring cells to occur without the necessity for many cells to rearrange in some mutually cooperative fashion that causes fluctuations to ripple across the monolayer. ${ }^{21}$ Because there are increasingly large regions over which cells have to move in a cooperative manner, rearrangements within cell packs must become increasingly cooperative, bigger and slower, as well as more intermittent. Such a remarkable growth in time scale and length scale manifests as a transition of the monolayer from a fluid-like to a solid-like state. A jammed system is said to be solid-like because, within the experimental time scale, it can resist applied stress by deforming elastically, as do coffee beans that become jammed in a chute, whereas an unjammed system will always flow. ${ }^{14,15,17,20,22,23}$ In inert soft condensed matter, similar behavior is called kinetic arrest.

\section{An analogy}

In the study of inert soft condensed matter, the observations of spontaneous intermittent fluctuations, dynamic heterogeneity, cooperativity, force chains, and kinetic arrest, when taken together, comprise the hallmarks of approach to a so-called glass transition that is thought to be associated with jamming..$^{20,22-27}$ Although jamming remains contentious and poorly understood, the concept has risen to prominence because it promises to unify understanding of a remarkably wide range of soft materials that include foams, pastes, colloids, slurries, suspensions, clays, and even in some instances granular matter like coffee beans in a chute, which can flow in some situations but jam in others.

The dynamics of the cellular collective comprising a monolayer is reminiscent of all these same hallmarks. Strikingly, these dynamics even conform quantitatively to the so-called Avramov-Milchev equation describing the rate of structural rearrangements ${ }^{17}$, and demonstrate growing scales of length and time as quantified using the more rigorous fourpoint susceptibility. ${ }^{15,28}$ Indeed, in both inert and living condensed systems, dynamics are 
constrained by many of the same physical factors, and, as such, the proposition of cell jamming might be not so unreasonable. For example, concerning the basic unit, whether a living cell, a foam bubble, a colloidal particle, or a coffee bean, these factors include volume exclusion (two particles cannot occupy the same space at the same time), volume (size) ${ }^{29}$, deformability ${ }^{30}$, mutual crowding, mutual caging ${ }^{31,32}$, mutual adhesion/repulsion ${ }^{24}$, and imposed mechanical deformation (stretch or shear). ${ }^{33-36} \mathrm{In}$ a monolayer, it is easy to imagine that cells might become freer to move as their size, crowding, stiffness or mutual adhesion become less, or as their motile forces or imposed stretch become more.

Conversely, it is easy to imagine that as adhesion or crowding progressively increases, or as motile forces progressively decrease, cellular rearrangements might become progressively slowed, cooperativity would increase, and, eventually, the monolayer would become topologically frozen and all cells would be caged by their neighbors. ${ }^{13,15,17-20}$ As such, it is reasonable to ask if the jamming hypothesis might unify within one mechanistic framework the effects of diverse biological factors previously considered to be acting more or less separately and independently.

\section{A speculation: The jamming phase diagram}

Generalizing from the literature of inert soft matter ${ }^{24,25}$, these effects in the living systems might be imagined to play out within a hypothetical jamming phase diagram (Fig. 5). In this diagram we represent on one axis cellular crowding, here expressed as the reciprocal of cellular density. In this manner, infinite cellular density is mapped to the origin. On another axis we represent cell-cell adhesion, again expressed as a reciprocal so that the case of infinitely sticky cells is again mapped to the origin. On yet another axis we map the effects of cell motile forces. Still other axes are certainly imaginable and probably necessary, but are not shown, such as imposed stretch or shear loading ${ }^{33,34}$, cellular volume ${ }^{29}$, cellular stiffness ${ }^{30}$, and substrate stiffness ${ }^{16,18}$. In this imagined multi-dimensional space, the origin, and regions near the origin, are necessarily jammed, and rearrangements are impossible because each cell is totally caged by its neighbors, or glued to its neighbors, or possesses no driving motile force. But away from the origin, especially along certain trajectories, structural rearrangements become increasingly possible. For example, stretch, apoptosis, or extrusion of cells from the monolayer ${ }^{37}$ would decrease cellular density, and as density becomes small enough the system might tend to unjam. Similarly, as adhesive interactions become small enough, or as stretch becomes large enough to disrupt adhesions, the system would be expected to unjam. And when motile forces become large enough, individual cells can pull away and dissociate from other cells, become loose and disaggregated and the system unjams.

If we re-examine the example of MCF10A cells through this lens we see that overexpression of ErbB2, which promotes proliferation and cell crowding, might be imagined to push the system even closer to a glassy and jammed state, whereas overexpression of 14-3-3 , which degrades cell-cell junctions, moves the system away from jamming and thereby fluidizes the system (Fig. 5). Another example would be scattering of MDCK cells induced by hepatocyte growth factor (HGF), which involves disruption of cadherindependent cell-cell junctions in a manner that is dependent upon integrin adhesion as well as phosphorylation of the myosin regulatory light chain. ${ }^{38}$ But these individual observations each fit nicely within this jamming phase diagram, with cells that are less jammed breaking from the pack to scatter.

\section{Bridging biology and physics}

Rather than just the binary possibilities of jammed vs. unjammed states, elaboration of the jamming phase diagram in inert systems demonstrate fragile intermediate states with 
potentially no less relevance to biology of the monolayer. ${ }^{22,23}$ If true, the jamming hypothesis would imply that specific events at the molecular scale necessarily modulate and respond to cooperative heterogeneities caused by jamming at a much larger scale of organization, but specific events at the molecular scale could never by themselves explain these cooperative large-scale events (Fig. 5). This perspective neither minimizes specific molecular events nor ignores them, but rather seeks to set them into an integrative framework that was unanticipated but might be overriding.

In the context of monolayer biology, the physical perspective of cell jamming leads logically to biological questions not previously considered. In physiology, does the epithelial monolayer tend to form a solid-like aggregated sheet- with excellent barrier function and with little possibility of cell invasion or escape- because constituent cells are jammed ${ }^{37}$ ? In pathophysiology, do certain cell populations become fluid-like and permissive of paracellular leak, transformation, cell escape or invasion because they become unjammed? Do pattern formation and wound healing require cell unjamming ${ }^{21}$ ? If so, what is the nature of the critical physical threshold? What are the signaling events and resulting physical changes that promote or prevent cell jamming? Conversely, at the level of gene expression and cell signaling, what are the signature effects of cell jamming? In this connection, forcedependent thresholds and novel pathways that control cell polarization have been recently reported ${ }^{3,39}$, but do these same thresholds and pathways pertain in collective processes? Moreover, human trials targeting adhesion molecules to slow tumor progression have proven to be ineffective, and this disappointment has been interpreted as reflecting that migration events are somehow reprogrammed- by mechanisms that remain undefined- so as to maintain invasiveness via morphological and functional dedifferentiation. ${ }^{2,40-42}$ Without excluding that possibility, might jamming allow for the alternative possibilities that certain tumor cell subpopulations may unjam, awake from dormancy and thus evolve so as to maintain invasiveness by selection for tradeoffs among adhesive interaction, compressive stress, and cyclic deformation? Each of these questions spans physics and biology and, with tools currently in hand, it is conceivable that these questions can now be broached.

\section{Acknowledgments}

The authors thank the Swiss National Science Foundation for financial support (grant PBEZP2_140047).

\section{Bibliography}

1. Friedl P, Alexander S. Cancer invasion and the microenvironment: plasticity and reciprocity. Cell. 2011; 147:992-1009. [PubMed: 22118458]

2. Friedl P, Gilmour D. Collective cell migration in morphogenesis, regeneration and cancer. Nat Rev Mol Cell Biol. 2009; 10:445-57. [PubMed: 19546857]

3. Weber G, Bjerke M, DeSimone D. A Mechanoresponsive Cadherin-Keratin Complex Directs Polarized Protrusive Behavior and Collective Cell Migration. Developmental Cell. 2012

4. Editors. News Focus: Mysteries of the Cell. Science. 2011; 334:1046-51.

5. Discher D, Dong C, Fredberg JJ, Guilak F, Ingber D, Janmey P, Kamm RD, Schmid-Schonbein GW, Weinbaum S. Biomechanics: cell research and applications for the next decade. Ann Biomed Eng. 2009; 37:847-59. [PubMed: 19259817]

6. Steinberg MS. Does differential adhesion govern self-assembly processes in histogenesis? Equilibrium configurations and the emergence of a hierarchy among populations of embryonic cells. J Exp Zool. 1970; 173:395-433. [PubMed: 5429514]

7. Foty RA, Steinberg MS. The differential adhesion hypothesis: a direct evaluation. Dev Biol. 2005; 278:255-63. [PubMed: 15649477]

8. Steinberg MS. Differential adhesion in morphogenesis: a modern view. Curr Opin Genet Dev. 2007; 17:281-6. [PubMed: 17624758] 
9. Wartlick O, Mumcu P, Kicheva A, Bittig T, Seum C, Julicher F, Gonzalez-Gaitan M. Dynamics of Dpp signaling and proliferation control. Science. 2011; 331:1154-9. [PubMed: 21385708]

10. Lauschke VM, Tsiairis CD, Francois P, Aulehla A. Scaling of embryonic patterning based on phase-gradient encoding. Nature. 2012

11. Keller R. Developmental biology. Physical biology returns to morphogenesis. Science. 2012; 338:201-3. [PubMed: 23066066]

12. D'Arcy Thompson, W. On Growth and Form. Cambridge University Press; 1942.

13. Ladoux B. Cells guided on their journey. Nature Physics. 2009; 5:377-8.

14. Trepat X, Fredberg JJ. Plithotaxis and emergent dynamics in collective cellular migration. Trends Cell Biol. 2011; 21:638-46. [PubMed: 21784638]

15. Tambe DT, Corey Hardin C, Angelini TE, Rajendran K, Park CY, Serra-Picamal X, Zhou EH, Zaman MH, Butler JP, Weitz DA, Fredberg JJ, Trepat X. Collective cell guidance by cooperative intercellular forces. Nature Materials. 2011; 10:469-75.

16. Krishnan R, Klumpers DD, Park CY, Rajendran K, Trepat X, van Bezu J, van Hinsbergh VW, Carman CV, Brain JD, Fredberg JJ, Butler JP, van Nieuw Amerongen GP. Substrate stiffening promotes endothelial monolayer disruption through enhanced physical forces. Am J Physiol Cell Physiol. 2011; 300:C146-54. [PubMed: 20861463]

17. Angelini TE, Hannezo E, Trepat X, Marquez M, Fredberg JJ, Weitz DA. Glass-like dynamics of collective cell migration. Proc Natl Acad Sci U S A. 2011; 108:4714-9. [PubMed: 21321233]

18. Angelini T, Hannezo E, Trepat X, Fredberg J, Weitz D. Cell Migration Driven by Cooperative Substrate Deformation Patterns. Physical Review Letters. 2010; 104:168104. [PubMed: 20482085]

19. Trepat X, Wasserman M, Angelini T, Millet E, Weitz D, Butler J, Fredberg J. Physical forces during collective cell migration. Nature Physics. 2009; 5:426-30.

20. Garrahan JP. Dynamic heterogeneity comes to life. Proc Natl Acad Sci U S A. 2011; 108:4701-2.

21. Serra-Picamal X, Conte V, Vincent R, Anon E, Tambe D, Bazellieres E, Butler J, Fredberg J, Trepat X. Mechanical waves during tissue expansion. Nature Physics. 2012; 8:628-34.

22. Bi D, Zhang J, Chakraborty B, Behringer RP. Jamming by shear. Nature. 2011; 480:355-8. [PubMed: 22170683]

23. Vitelli V, van Hecke M. Soft materials: Marginal matters. Nature. 2011; 480:325-6. [PubMed: 22170675]

24. Trappe V, Prasad V, Cipelletti L, Segre PN, Weitz DA. Jamming phase diagram for attractive particles. Nature. 2001; 411:772-5. [PubMed: 11459050]

25. Liu AJ, Nagel SR. Jamming is not just cool any more. Nature. 1998; 396:21-2.

26. Liu CH, Nagel SR, Schecter DA, Coppersmith SN, Majumdar S, Narayan O, Witten TA. Force Fluctuations in Bead Packs. Science. 1995; 269:513-5. [PubMed: 17842361]

27. Lu K, Brodsky E, Kavehpour H. A thermodynamic unification of jamming. Nature Physics. 2008

28. Berthier L, Biroli G, Bouchaud JP, Cipelletti L, El Masri D, L'Hote D, Ladieu F, Pierno M. Direct experimental evidence of a growing length scale accompanying the glass transition. Science. 2005; 310:1797-800. [PubMed: 16357256]

29. Zhou EH, Trepat X, Park CY, Lenormand G, Oliver MN, Mijailovich SM, Hardin C, Weitz DA, Butler JP, Fredberg JJ. Universal behavior of the osmotically compressed cell and its analogy to the colloidal glass transition. Proc Natl Acad Sci U S A. 2009; 106:10632-7. [PubMed: 19520830]

30. Mattsson J, Wyss HM, Fernandez-Nieves A, Miyazaki K, Hu Z, Reichman DR, Weitz DA. Soft colloids make strong glasses. Nature. 2009; 462:83-6. [PubMed: 19890327]

31. Schall P, Weitz DA, Spaepen F. Structural rearrangements that govern flow in colloidal glasses. Science. 2007; 318:1895-9. [PubMed: 18096800]

32. Segre PN, Prasad V, Schofield AB, Weitz DA. Glasslike kinetic arrest at the colloidal-gelation transition. Phys Rev Lett. 2001; 86:6042-5. [PubMed: 11415424]

33. Trepat X, Deng L, An S, Navajas D, Tschumperlin D, Gerthoffer W, Butler J, Fredberg J. Universal physical responses to stretch in the living cell. Nature. 2007; 447:592-5. [PubMed: 17538621] 
34. Krishnan R, Park CY, Lin YC, Mead J, Jaspers RT, Trepat X, Lenormand G, Tambe D, Smolensky AV, Knoll AH, Butler JP, Fredberg JJ. Reinforcement versus fluidization in cytoskeletal mechanoresponsiveness. PLoS ONE. 2009; 4:e5486. [PubMed: 19424501]

35. Oliver M, Kova'ts T, Mijailovich S, Butler J, Fredberg J, Lenormand G. Remodeling of Integrated Contractile Tissues and Its Dependence on Strain-Rate Amplitude. Physical Review Letters. 2010; 105:158102. [PubMed: 21230941]

36. Wyss HM, Miyazaki K, Mattsson J, Hu Z, Reichman DR, Weitz DA. Strain-rate frequency superposition: a rheological probe of structural relaxation in soft materials. Phys Rev Lett. 2007; 98:238303. [PubMed: 17677943]

37. Eisenhoffer GT, Loftus PD, Yoshigi M, Otsuna H, Chien CB, Morcos PA, Rosenblatt J. Crowding induces live cell extrusion to maintain homeostatic cell numbers in epithelia. Nature. 2012; 484:546-9. [PubMed: 22504183]

38. de Rooij J, Kerstens A, Danuser G, Schwartz MA, Waterman-Storer CM. Integrin-dependent actomyosin contraction regulates epithelial cell scattering. J Cell Biol. 2005; 171:153-64. [PubMed: 16216928]

39. Prager-Khoutorsky M, Lichtenstein A, Krishnan R, Rajendran K, Mayo A, Kam Z, Geiger B, Bershadsky AD. Fibroblast polarization is a matrix-rigidity-dependent process controlled by focal adhesion mechanosensing. Nat Cell Biol. 2011; 13:1457-65. [PubMed: 22081092]

40. Friedl P, Hegerfeldt Y, Tusch M. Collective cell migration in morphogenesis and cancer. Int J Dev Biol. 2004; 48:441-9. [PubMed: 15349818]

41. Friedl P, Wolf K. Tumour-cell invasion and migration: diversity and escape mechanisms. Nat Rev Cancer. 2003; 3:362-74. [PubMed: 12724734]

42. Rice J. The rude awakening. Nature. 2012; 485:S55-7. [PubMed: 22648500] 


\section{Research Highlights}

- Wound healing, development, and invasion are collective cellular processes.

- Collectivity depends upon factors including crowding, adhesion, and contractility.

- These and other factors may be unified by the concept of cell jamming.

- The jamming concept leads to interesting insights and provocative new questions. 

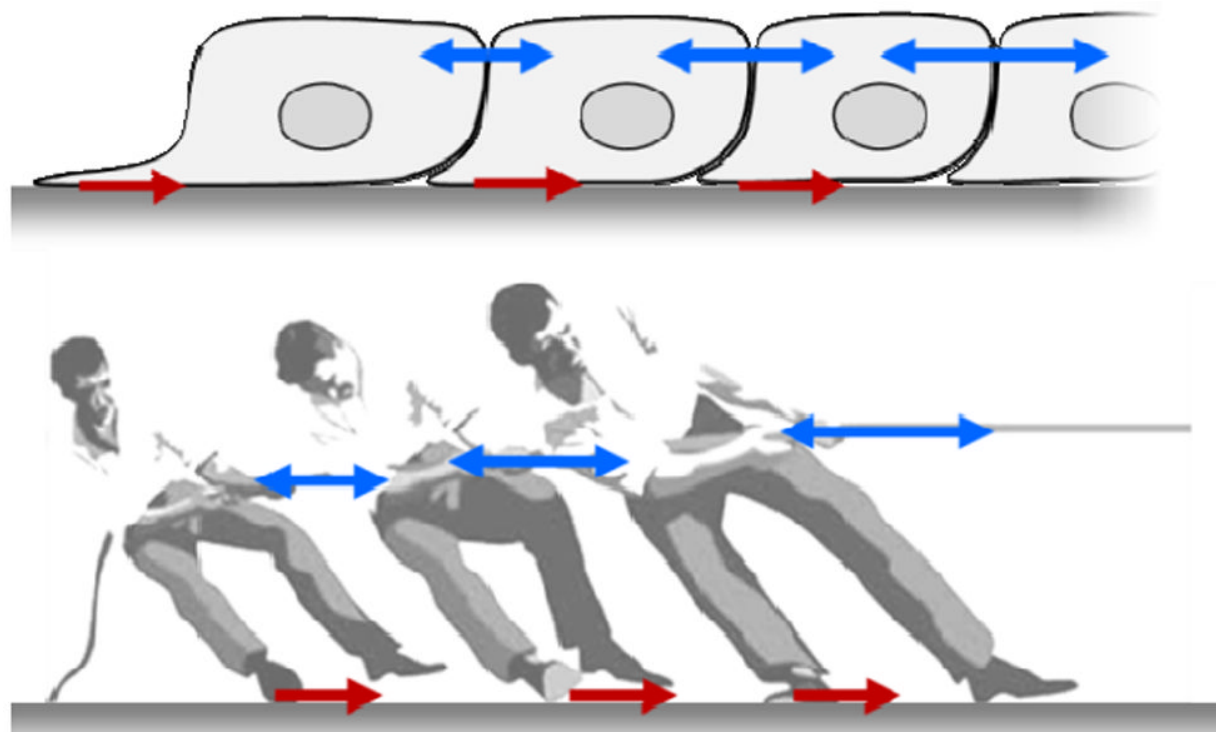

Fig. 1. Cells use a tug-of-war mechanism to integrate local tractions (red) into long-range gradients of intra- and inter-cellular tension (blue)

Tension in the monolayer reflects the spatial accumulation, or pile-up of traction forces. Equivalently, the local traction force is the spatial derivative of the intercellular stress. Reprinted with permission from ${ }^{14}$ 


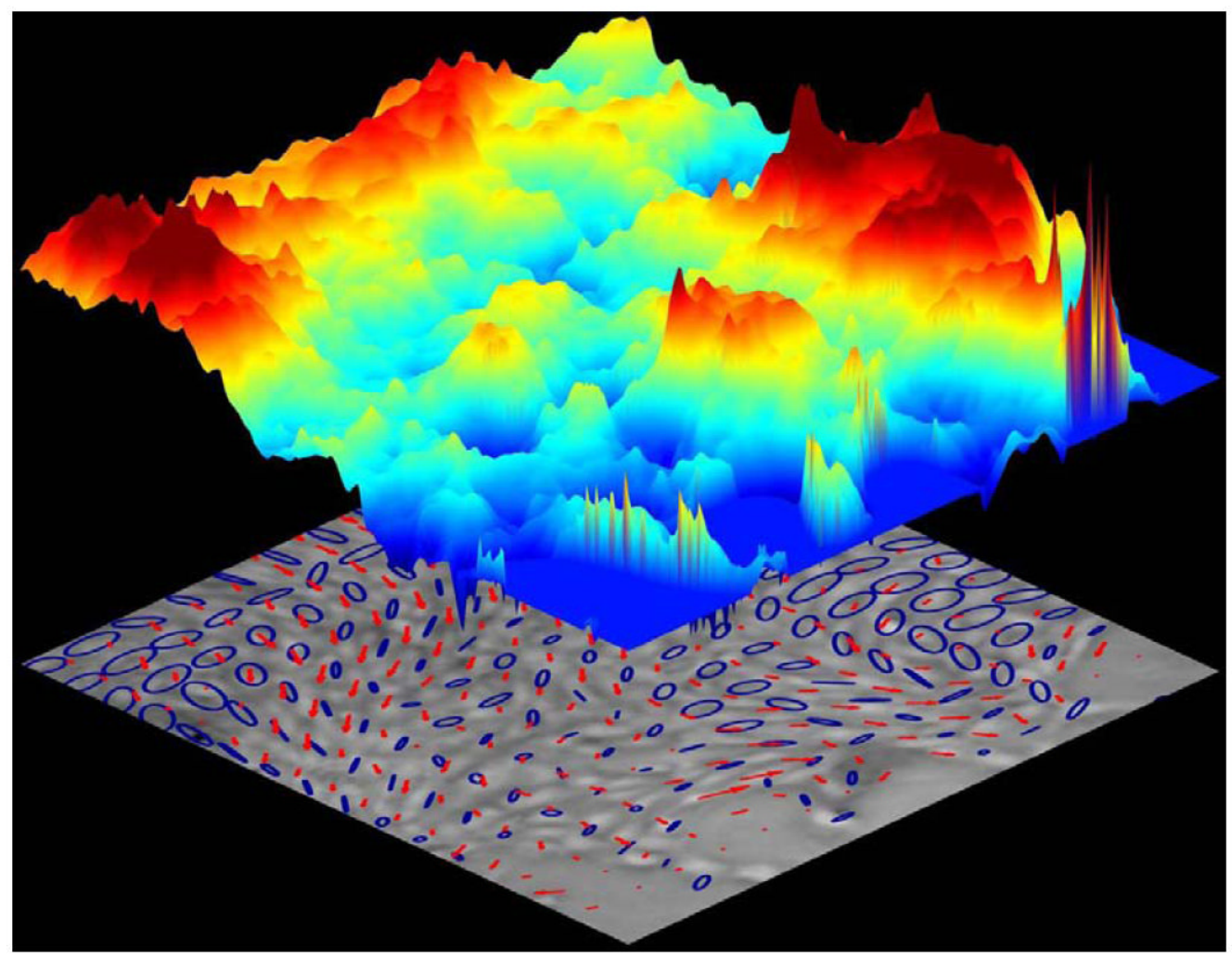

Fig 2. Malleable cells trek a rugged stress landscape and make for a resilient monolayer Cellular migrations (red arrows) follow stress orientations (blue ellipses) over a rugged stress landscape (colored topography denotes local tensile stress; scale bar units: Pa). Cell navigation on this scale- plithotaxis- is innately collective, strongly cooperative, and dynamically glassy. Reprinted with permission from 15 

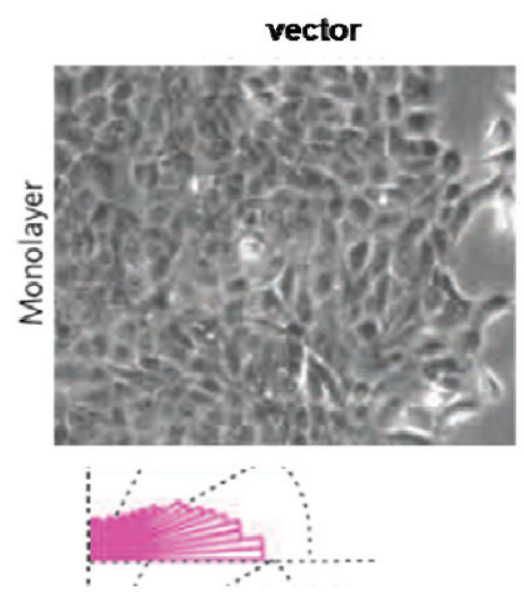

Overexpress ErbE2
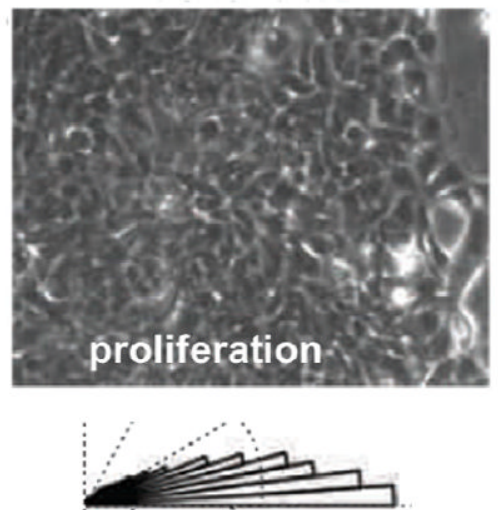

Plithotaxis strong Strong cell-cell guidance
Overexpress 14 $14-35$
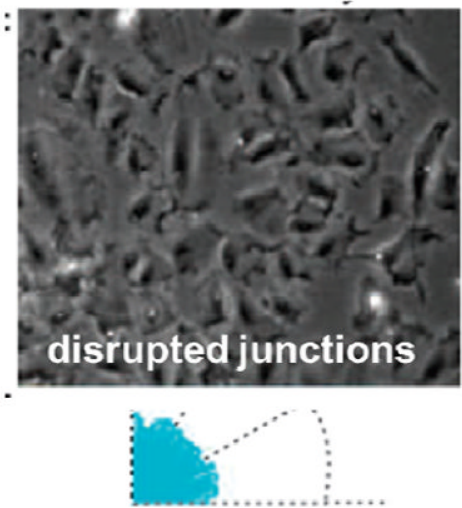

Plithotaxis ablated No cell-cell guidance

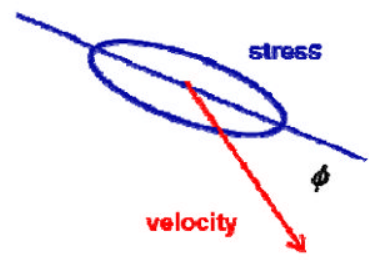

Fig. 3. Stress maps and migration in monolayers of breast-cancer model systems Phase contrast image of nontransformed human mammary epithelial cell line, MCF10A, control or vector, cells overexpressing ErbB2, and 14-3-3ろ. The angle $\varphi$ defines the orientation between the maximal principle stress and the migration velocity. In each case, the histogram of $\varphi$ is plotted as a rose of directions. Adapted with permission from ${ }^{15}$. 

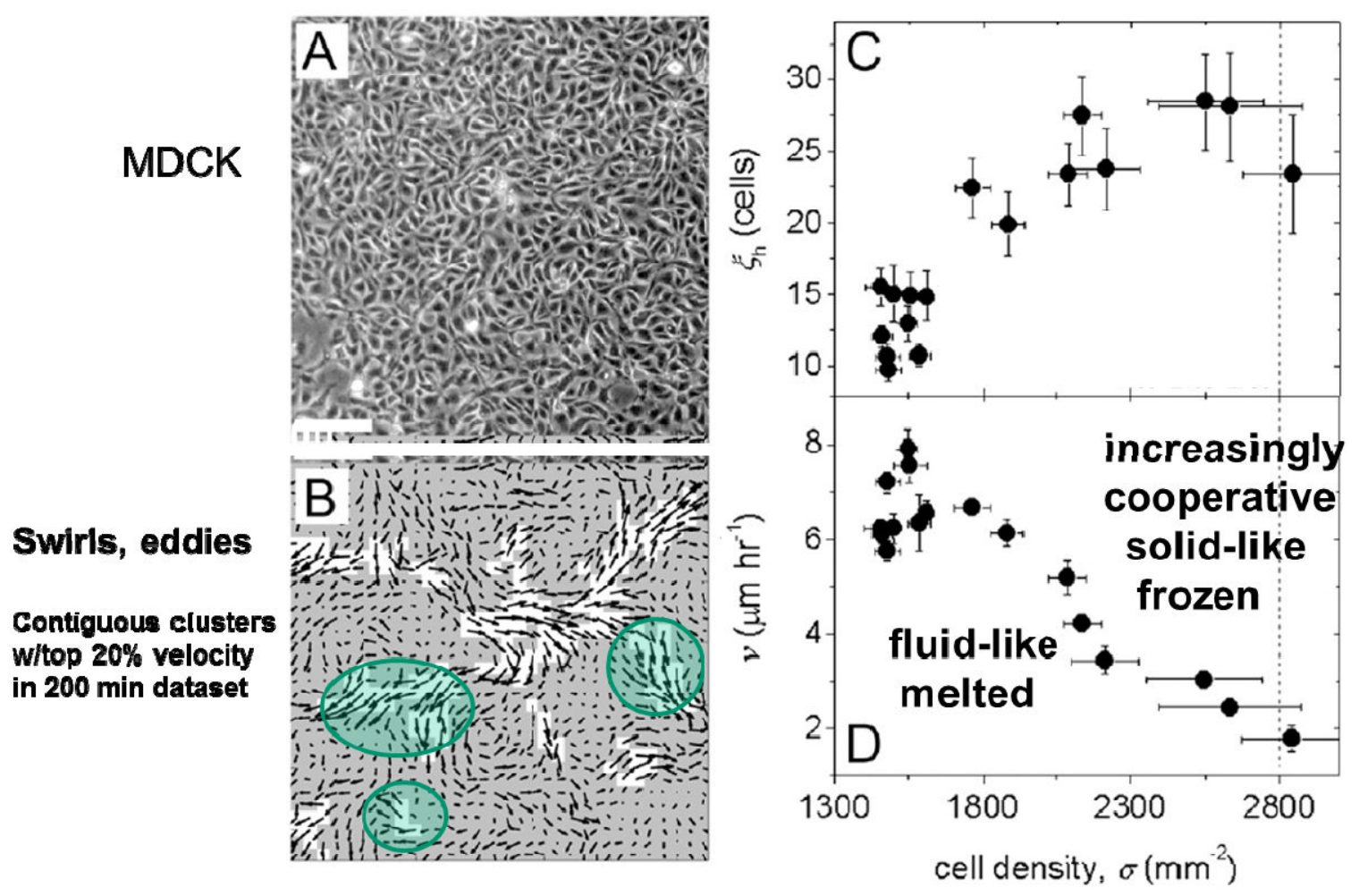

Fig. 4. MDCK cells within a confluent monolayer migrate in a spatially heterogeneous manner $(\mathrm{A}, \mathrm{B})$

The average area of contiguous regions of the fastest velocity vectors defines $\xi_{\mathrm{h}}$, the area of dynamic heterogeneities ( $\mathrm{B}$, white regions). As cell density rises, $\boldsymbol{\xi}_{\mathrm{h}}$ grows from an area of about 10 cell bodies to 30 cell bodies $\left(\mathrm{C}\right.$, inset: $\xi_{\mathrm{h}}$ in $\left.\mu \mathrm{m}^{2}\right)$. The average migration speed of cells within the entire field of view, $v$, decreases with increasing cell density (D). (Scale bar, $100 \mu \mathrm{m}$.). Reprinted with permission from 17 


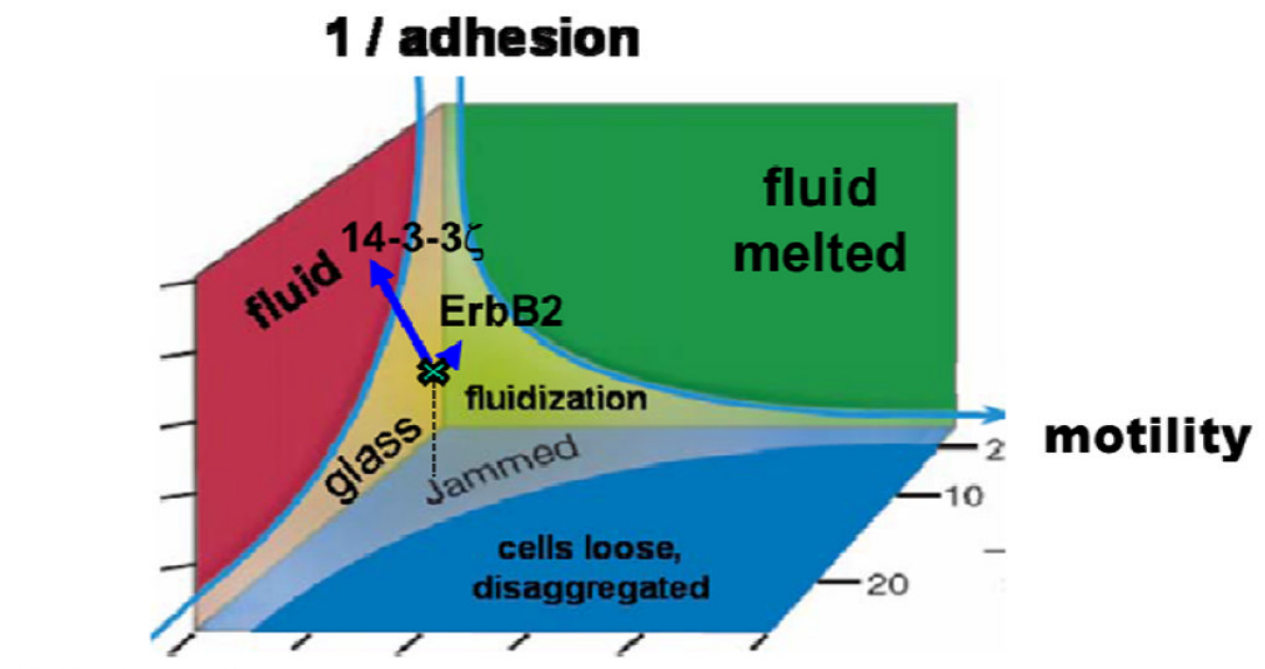

\section{$1 /$ density}

Fig. 5. Hypothetical jamming phase diagram for the cellular monolayer

As cells express more mutual crowding, more mutual adhesion, or less myosin-dependent motile force, associated coordinates in phase space move progressively closer to the origin and the monolayer state becomes increasingly jammed. Transition toward a jammed state and resulting glassy dynamics is depicted by the hypothetical shaded surface. Arrow-heads depict the migration speed and migration direction of individual cells. Colors depict cell clusters (packs) that move collectively. Vector: Cells in the control state (MCF10A-vector) exist close to but just outside the jamming transition, where cells move in packs that become progressively larger and slower as the jamming transition is approached. ${ }^{15,17,18}$ Local orientation of cell migration correspond closely to the local orientation of maximal principal stress (not shown); this mechanism of cell collective guidance is called plithotaxis. ${ }^{14,15}$

Overexpression of oncogene ErbB2: As cells proliferate and crowd more densely, cell packs become progressively larger and slower, and plithotaxis becomes amplified.

Overexpression of oncogene 14-3-3ろ: As cell-cell junctions lose cadherin-dependent adhesion, the monolayer becomes fluidized and unjammed. Collectivity is lost and plithotaxis is ablated. Adapted from 24,25 